\title{
Ethanol inactivation of orthonairoviruses in ixodid ticks
}

\author{
A. Schulz ${ }^{1} \cdot$ K. Methling ${ }^{2} \cdot$ M. Lalk ${ }^{2} \cdot$ A. Eisenbarth ${ }^{3} \cdot$ M. Keller ${ }^{1} \cdot$ M. H. Groschup ${ }^{1}$
}

Received: 10 March 2021 / Accepted: 1 September 2021 / Published online: 20 September 2021

(c) The Author(s) 2021

\begin{abstract}
Ixodid ticks represent vectors and reservoirs for a broad range of zoonotic pathogens. Collected ticks from field studies are therefore usually stored in ethanol, which in higher concentrations effectively inactivates most of the known tick-borne pathogens. Although commonly practiced as gold standard for inactivation, hardly any scientific data demonstrate that ethanol sufficiently penetrates the comparatively thick cuticula of ticks. Therefore, Amblyomma hebraeum tick pools were stored for 21 days in ethanol (96\%). Afterwards, the ethanol was removed and the ticks were homogenized. Quantitative ${ }^{1} \mathrm{H}-\mathrm{NMR}$ spectroscopic analysis was applied to determine the residual concentration of ethanol inside the ticks. ${ }^{1} \mathrm{H}-\mathrm{NMR}$ spectroscopic analysis revealed that ethanol constituted $28.3-42.6 \mathrm{mg}$ of the total weight of three ticks in the pools (89.9-121.5 mg). In addition, the low-pathogenic Hazara orthonairovirus (HAZV) was used as a cell culture model for this study. The virus was exposed to ethanol concentrations between 0 and $60 \%$ and incubated under various temperature conditions for four time periods. Afterwards, the residual virus infectivity was determined by titration. Following ethanol exposure, HAZV did not grow in cells after $9 \mathrm{~h}$ of exposure to an ethanol concentration of $25 \%$. These results demonstrate an extremely low ethanol resistance of the virus, which was generally in line with previously reported ethanol inactivation data for Crimean-Congo hemorrhagic fever orthonairovirus (CCHFV). After prolonged storage and impregnation, comparable ethanol concentrations are achieved in the ticks, indicating the suitability of this inactivation method also for Bunyaviruses in ticks. At the very least, a massive virus inactivation can be assumed. Definitive proof of virus inactivation would require a bioassay of ethanol-treated infected ticks under appropriate biosafety conditions.
\end{abstract}

Keywords Ticks · Inactivation · Ethanol · Tick-borne pathogens $\cdot$ Quantitative ${ }^{1} \mathrm{H}-\mathrm{NMR}$ spectroscopy

M. H. Groschup

martin.groschup@fli.de

1 Friedrich-Loeffler-Institut, Institute of Novel and Emerging Infectious Diseases, Südufer 10, 17493 Greifswald-Insel Riems, Germany

2 Institute of Biochemistry, University of Greifswald, Felix-Hausdorff-Straße 4, 17489 Greifswald, Germany

3 Bundeswehrkrankenhaus Hamburg, Abt. XXI, Außenstelle BNITM, Bernhard-Nocht-Str. 74, 20359 Hamburg, Germany 


\section{Introduction}

Ixodid ticks are vectors and reservoirs for a broad variety of highly pathogenic zoonotic bacteria and viruses potentially causing life-threatening diseases (bacteria: Anaplasmosis, Babesiosis, Borreliosis, etc.; viruses: Tick borne Encephalitis, Omsk hemorrhagic fever, Crimean-Congo hemorrhagic fever, Severe Febrile Thrombocytopenia Syndrome, etc. (for a more extensive list, see https://www.cdc.gov/ticks/diseases/index.html). One of the most grievous tick-borne viruses, classified as biosafety level 4 pathogen, is Crimean-Congo hemorrhagic fever orthonairovirus (CCHFV). This virus belongs to the Nairovididae family in the Orthonairovirus genus and is transmitted by ticks of the genus Hyalomma, being its main vector and reservoir (Bente et al. 2013; Gargili et al. 2017). Humans can develop severe disease symptoms including fever, nausea and hemorrhagic bleedings with lethality rates of up to 30\% (Thangamani and Bente 2014; Whitehouse 2004). Studying and diagnostic screening of ixodid ticks and their zoonotic pathogens has therefore become an essential part of modern public health research. However, in order to safely handle and taxonomically identify tick species possibly infected with CCHFV at a lower biosafety level, the virus must therefore be reliably inactivated.

For this purpose and for their preservation, ticks are usually stored in ethanol, which at higher concentrations effectively inactivates most of the known tick-borne pathogens over time. While the inactivation kinetics for individual pathogens can be deduced from in vitro cell culture studies (Kampf 2018), there are hardly any, if at all, scientific data available on the actual rate of penetration of ethanol into the tissue of ticks. Diffusion of the small polar ethanol molecules is likely, but it may also be hindered by the strong chitin cuticle ixodid ticks possess, which is composed of a polysaccharide matrix of $\mathrm{N}$-acetylglucosamine units, crosslinked together with proteins, and of lipids. Depending on the protein types and the density and thickness of chitin, the cuticular exoskeleton can be extremely durable and solid. However, this hull is penetrated by orifices such as the mouth stomata, the spiracles and the genital as well as anal pores. Furthermore, the cuticle shields are, as common with all arthropods, connected and therefore interrupted by junctions and joints. Hence, it is plausible that the ethanol penetrates preferentially along these routes, but data on ethanol concentrations inside ethanol immersed ticks have never been determined properly.

Therefore, the main objective of the study was to determine the ethanol concentration in ticks after a defined submersion time in $96 \%$ alcohol. As this study was primarily motivated in order to facilitate working with $\mathrm{CCHFV}$, we screened the literature for reports on the alcohol resistance of this virus. A previous study showed that CCHFV was already undetectable after 2 min in 30\% ethanol using an in vitro cell culture system (Hardestam et al. 2007). We therefore determined the ethanol stability of Hazara orthonairovirus (HAZV), which is a very closely related but non-pathogenic relative of CCHFV, in a similar cell culture system. The goal of the study was to prove that (1) ethanol does indeed diffuse into the tick body and (2) thereby accumulates in internal concentrations suitable for virus inactivation. 


\section{Materials and methods}

\section{Measurement of ethanol in ixodid ticks}

In the absence of a Hyalomma tick colony, Ambylomma (A.) hebraeum ticks, originating from a laboratory colony (kindly provided by MSD Animal Health Innovation, Schwabenheim, Germany) were used as another ixodid tick species for evaluating the permeability of ethanol into a tick corpus. This tropical hard tick is well suited as a model, as it is a very large species similar to most Hyalomma spp. ticks.

Seven tick pools were stored for 21 days at room temperature in $1.5 \mathrm{ml}$ absolute ethanol (96\%) using 2-ml Eppendorf tubes. Each tick pool consisted of three adult A. hebraeum specimens (unfed) of the same sex. Overall, four pools of males (12 ticks in total) and three pools of females (nine ticks in total) were examined. After 21 days of exposure, the ticks were removed from the ethanol and rinsed with distilled water. Exceeding water was wiped off with absorbent paper towel. Subsequently, the tick pools were homogenized in $1 \mathrm{ml}$ of distilled water using a 5-mm-diameter steel bead. The weight of the samples was determined by precision balance at all crucial stages of the procedure. After centrifugation (10,000 rpm; $20 \mathrm{~min}), 500 \mu \mathrm{l}$ supernatant was used for the ${ }^{1} \mathrm{H}-\mathrm{NMR}$ spectroscopy-based quantification of ethanol. Distilled water was used as negative control.

\section{${ }^{1} \mathrm{H}$-NMR spectroscopy-based quantification of ethanol}

${ }^{1} \mathrm{H}-\mathrm{NMR}$ analysis and quantification were performed as previously described with further modifications (Dorries and Lalk 2013). Samples were diluted 1:100 in pure water (HPLC-grade) and analyses were done in 5-mm-diameter glass tubes (103.5 mm long; Bruker Biospin, Rheinstetten, Germany). The Bruker AVANCE-NEO 600 NMR spectrometer equipped with a SampleJet autosampler and a 5-mm QCI cryo probe was operated by TOPSPIN v.4.0.6 software (Bruker Biospin). Quantification was done using AMIX software v.3.9.15 by integration and comparison of triplet peak of ethanol at $1.186 \mathrm{ppm}$ to the ERETIC signal which was generated by using external calibration with the ERETIC quantification tool based on PULCON (Wider and Dreier 2006).

\section{Ethanol virus inactivation in cell culture}

Hazara orthonairovirus (HAZV) was used as a model orthonairovirus for this study. HAZV was grown SW-13 cells with L-15 (Leibovitz) medium (Sigma-Aldrich, St. Louis, MO, USA) supplemented with $100 \mathrm{U} / \mathrm{ml}$ penicillin (Sigma-Aldrich), $0.1 \mathrm{mg} / \mathrm{ml}$ streptomycin (Sigma-Aldrich) and 2\% fetal bovine serum. The virus was harvested $48 \mathrm{~h}$ post infection and the tissue culture infectious dose $\left(\mathrm{TCID}_{50}\right)$ was calculated by the Spearman-Karber method. In order to create conditions similar to blood-fed infected ticks from the field and thus account for the possible influence of host blood, the virus-containing cell culture supernatant (10\%) was mixed with bovine serum (80\%) as well as bovine EDTA (10\%). In this mixture, the virus was exposed to concentrations of $0-60 \%$ ethanol using a dilution series. The blood/virus mixture was eventually incubated under various temperature conditions $\left(-20{ }^{\circ} \mathrm{C}, 4^{\circ} \mathrm{C}\right.$ or room temperature) for four time periods $(0,3,9,24 \mathrm{~h})$. Afterwards, the residual virus infectivity was determined by titration on SW-13 cells (6-well plates). 
Due to the cytotoxicity of ethanol, the virus cultivation at 6-well plates was conducted in an additional 1:20 dilution using the same media as described above. All samples were cultured in duplicate including positive and negative controls.

\section{Results}

\section{Ethanol concentration in ticks}

${ }^{1} \mathrm{H}-\mathrm{NMR}$ spectroscopic analysis revealed that the tick pools $(1000 \mu \mathrm{l})$ contained 28.3-42.6 mg pure ethanol (Table 1). As a result, ethanol constituted 31-37.7\% of the total weight of three ticks each per pool (89.9-121.5 mg) after 3 weeks of storage in ethanol. No ethanol was found in the negative control.

\section{Virus titration and cultivation under the influence of ethanol}

The initial HAZV concentration in the cell culture supernatant was $10^{7.8} / \mathrm{ml} \mathrm{TCID}_{50}$. Following ethanol exposure, a significant virus reduction started already at concentrations above $15 \%$ and a complete inactivation was observed with $20 \%$ ethanol (Fig. 1). It should be noted that ethanol concentrations above $25 \%$ were toxic to the cells. However, HAZV did not grow anymore in SW-13 cells after $9 \mathrm{~h}$ of exposure to an ethanol concentration of $25 \%$.

\section{Discussion}

In recent decades, the risk of emerging viral, bacterial and parasitic infectious diseases and their spread has risen considerably in response to globalization, climatic changes and increased human intervention in intact ecosystems. Studies have shown that tropical ticks and mosquito species are spreading continuously into previously non-endemic areas, thus increasing the risk of permanent introduction of (neglected) tropical diseases into these areas (Epstein 2001). Therefore, screening of tick vectors for pathogens in order to understand their occurrence and emergence has become an essential part of modern epidemiological research. In this context, a reliable inactivation of all potential infectious agents inside the ticks is crucial to ensure safe working conditions for the laboratory personnel in terms of biosafety / biosecurity.

Table 1 Ethanol weight in relation to the total weight of the ticks after 3 weeks storage in $96 \%$ ethanol

\begin{tabular}{lllll}
\hline Tick sex & Pool nr. & $\begin{array}{l}\text { Ticks weight } \\
(\mathrm{mg})\end{array}$ & $\begin{array}{l}\text { Ethanol } \\
\text { weight }(\mathrm{mg})\end{array}$ & Ratio (\%) \\
\hline Female & 1 & 98.1 & 30.4 & 31.0 \\
& 2 & 81.9 & 30.9 & 37.7 \\
& 3 & 121.5 & 42.6 & 35.0 \\
Male & 4 & 108.5 & 36.2 & 33.3 \\
& 5 & 92.9 & 32.9 & 35.5 \\
& 6 & 107.3 & 38.0 & 35.5 \\
& 7 & 82.1 & 28.3 & 34.5 \\
\hline
\end{tabular}




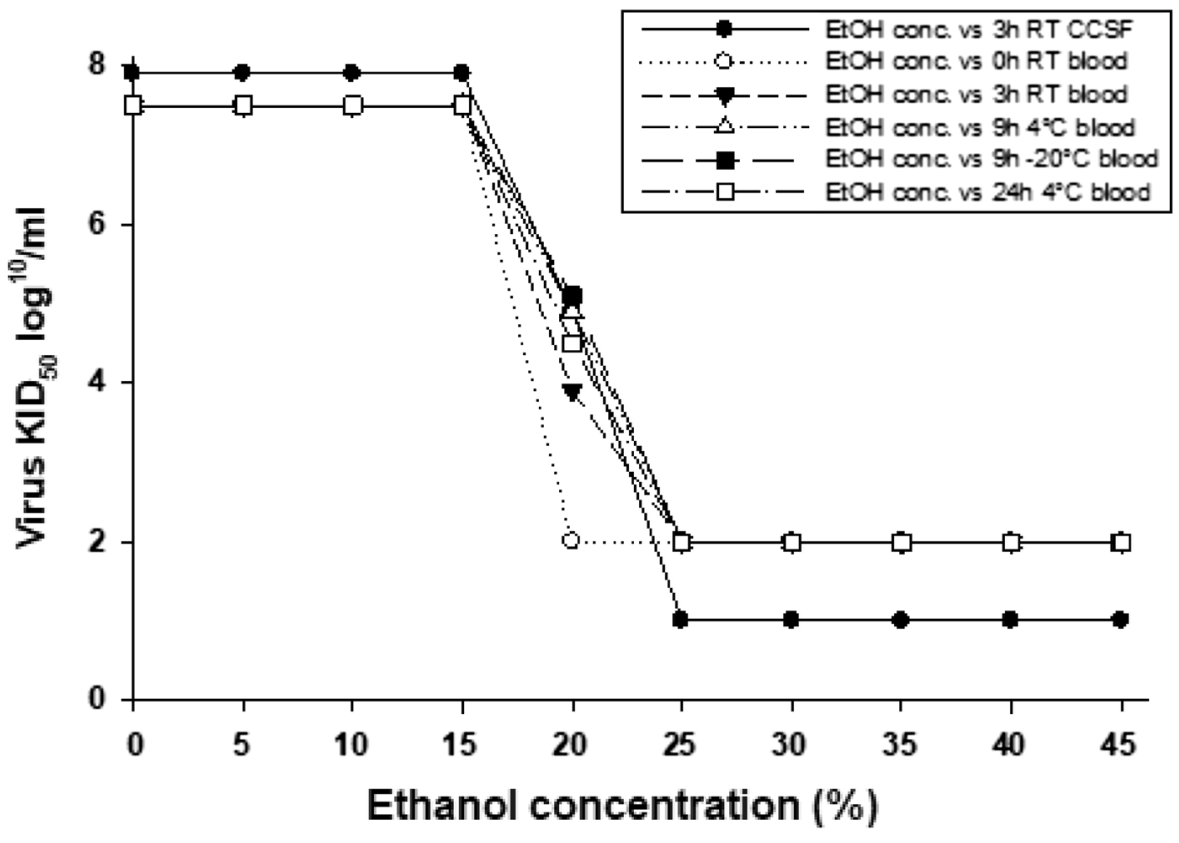

Fig. 1 Titration results of Hazara orthonairovirus in various ethanol concentrations, at three temperatures ( $R T$ room temperature; $E t O H$ conc., ethanol concentration; $C C S F$ cell culture supernatant fluid). The figure was created with SigmaPlot v.11

The presented HAZV ethanol inactivation study results were generally in line with previously reported data (Hardestam et al. 2007) in which both CCHFV and HAZV infectivity was not detectable after remarkably short exposure times to 30 and $25 \%$ ethanol, respectively. Our results demonstrate that such concentrations are easily reached during a 3 -week storage of unfed ticks in ethanol (96\%). According to the quantitative ${ }^{1} \mathrm{H}-\mathrm{NMR}$ spectroscopic data, ethanol constitutes about one third of the mass of the examined tick pools (31.0-37.7\%). The exact organ distribution of the alcohol inside the ticks or the actual dilution rate of the $96 \%$ ethanol cannot be calculated directly based on these data. However, due to the highly hydrophilic character of ethanol, it can be assumed that it will diffuse evenly into all aqueous compartments within the tick body, including the salivary glands and gastrointestinal tract. Nevertheless, the best proof of virus inactivation would be achieved by an in vivo inactivation study that should involve an experimental infection of ticks of varying feeding levels, the storage in ethanol and a re-isolation attempt of the virus.

\section{Conclusion}

The obtained results indicate a suitably high ethanol diffusion into ticks to inactivate HAZV and CCHFV inside. Therefore, these data provide a proof of principle regarding the choice of adequate biosafety standards for laboratory work with ticks potentially harboring tick-borne pathogens. 
Acknowledgements We thank René Schöttner for his excellent technical support and performing the experiments. We also thank Cornelia Silaghi and MSD Animal Health Innovation, Schwabenheim, Germany for providing the ticks from the laboratory colony.

Author contributions AS: conceptualization, methodology, data curation and validation, writing of first draft. KM: practical implementation, data curation, writing (review \& editing). ML: conceptualization, writing (review \& editing). AE: conceptualization, methodology, writing (review \& editing). MK: methodology, writing (review \& editing). MHG: design of research strategy and overall supervision of its implementation, data curation and validation, resources and funding, writing (review \& editing).

Funding Open Access funding enabled and organized by Projekt DEAL. This study was co-funded by the German Office for Foreign Affairs (German Partnership Program for Biosecurity) and by the Deutsche Forschungsgemeinschaft (Grant No. GR980/4-1 AOBJ 630130) and the Mecklenburg-Pomerania Excellence Initiative (Germany) and European Social Fund (ESF) Grant KoInfekt (Grant No. ESF_14-BM-A55-0005_16).

Data availability All data generated or analyzed during this study are included in this published article.

\section{Declarations}

Conflict of interest There were no competing interests among the authors.

Open Access This article is licensed under a Creative Commons Attribution 4.0 International License, which permits use, sharing, adaptation, distribution and reproduction in any medium or format, as long as you give appropriate credit to the original author(s) and the source, provide a link to the Creative Commons licence, and indicate if changes were made. The images or other third party material in this article are included in the article's Creative Commons licence, unless indicated otherwise in a credit line to the material. If material is not included in the article's Creative Commons licence and your intended use is not permitted by statutory regulation or exceeds the permitted use, you will need to obtain permission directly from the copyright holder. To view a copy of this licence, visit http://creativecommons.org/licenses/by/4.0/.

\section{References}

Bente DA, Forrester NL, Watts DM, McAuley AJ, Whitehouse CA, Bray M (2013) Crimean-congo hemorrhagic fever: history, epidemiology, pathogenesis, clinical syndrome and genetic diversity. Antiviral Res 100:159-189. https://doi.org/10.1016/j.antiviral.2013.07.006

Dorries K, Lalk M (2013) Metabolic footprint analysis uncovers strain specific overflow metabolism and D-isoleucine production of Staphylococcus aureus col and hg001. PLoS ONE 8:e81500. https://doi. org/10.1371/journal.pone.0081500

Epstein PR (2001) Climate change and emerging infectious diseases. Microb Infect 3:747-754. https://doi. org/10.1016/s1286-4579(01)01429-0

Gargili A, Estrada-Pena A, Spengler JR, Lukashev A, Nuttall PA, Bente DA (2017) The role of ticks in the maintenance and transmission of crimean-congo hemorrhagic fever virus: a review of published field and laboratory studies. Antiviral Res 144:93-119. https://doi.org/10.1016/j.antiviral.2017.05.010

Hardestam J, Simon M, Hedlund KO, Vaheri A, Klingström J, Lundkvist A (2007) Ex vivo stability of the rodent-borne hantaan virus in comparison to that of arthropod-borne members of the Bunyaviridae family. Appl Environ Microbiol 73:2547-2551. https://doi.org/10.1128/aem.02869-06

Kampf G (2018) Efficacy of ethanol against viruses in hand disinfection. J Hosp Infect 98:331-338. https:// doi.org/10.1016/j.jhin.2017.08.025

Thangamani S, Bente D (2014) Establishing protocols for tick containment at biosafety level 4. Pathog Dis 71:282-285. https://doi.org/10.1111/2049-632X.12187

Whitehouse CA (2004) Crimean-congo hemorrhagic fever. Antiviral Res 64:145-160. https://doi.org/10. 1016/j.antiviral.2004.08.001

Wider G, Dreier L (2006) Measuring protein concentrations by nmr spectroscopy. J Am Chem Soc 128:2571-2576. https://doi.org/10.1021/ja055336t 
Publisher's Note Springer Nature remains neutral with regard to jurisdictional claims in published maps and institutional affiliations. 